Health \& Medicine | Richard B. Aguilar

\section{COVID-19}

An individualized treatment framework

The elderly are among those at greatest risk of serious infection and death from the coronavirus (COVID-19). By individualizing treatment for COVID-19 and its complications, a significant advance in medicin and improving care could be and colleagues have developed the first known evidencebased recommendations individualized treatment. These recommendations were derived from a preliminary review of existing research, followed by implementation of a disease progression and treatment model from a cohort key to improving outcomes is determining risk and individualizing care. The tailoring of COVID-19 management is in its nascent form. evidence-based recommendations for individualized treatment of COVID-19 and subsequently reported on its application in a primary care setting. They demonstrated a significant reduction in hospitalization and mortality from COVID-19 on their patient base. The team have been contributing to these efforts with their large cohort of primarily elderly and evaluation of individualized care protocols. This was done across their clinics, and included those patients at greatest risk of severe complications.

Early during the COVID-19 pandemic, the team applied their clinical and population health tools to attempt to bend the curve and improve clinical outcomes. Their initial publication in October 2020, Current Understanding of COVD-19 Clinical Course and Investigational Treatments, proposed a treatmen algorithm for patients infected with COVID-19. They put forth the concep that a treatment algorithm should be dependent on vanying factors such as age, gender, com

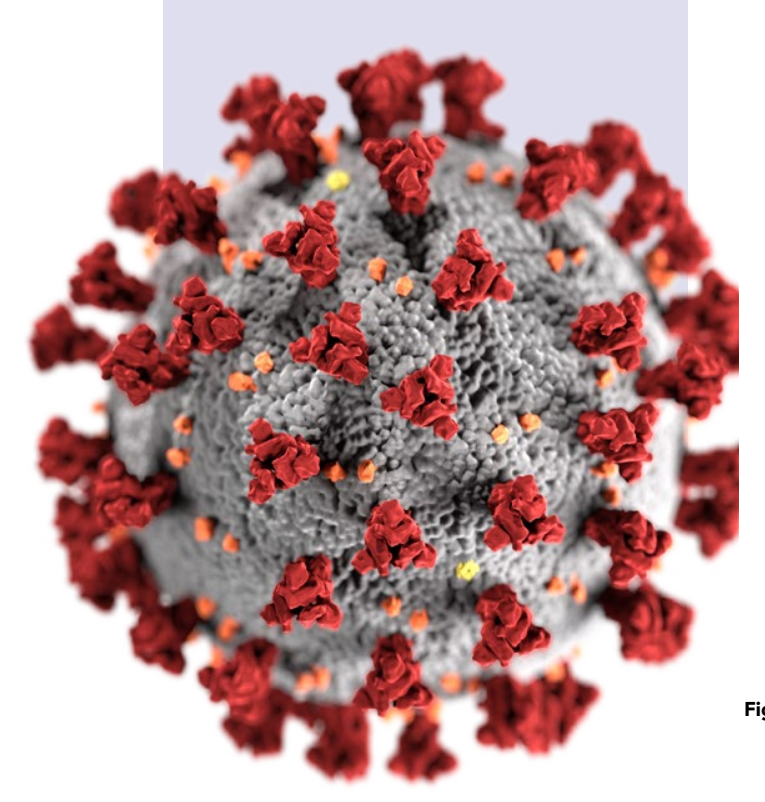

After applying these treatment principles Adver 38,000 of their Medicare (a) COVID-19 Outcomes in a Popultion Health Program, comparing their results to an age- and gender-matched musuls population in Florida (SOF).

Their findings supported their hypothesis that managed care patients would fare better than a comparable population primarily due to their model of care. This model of care proactively focused on keeping patients healthy rather than being reactionary and just treating disease. Their papers concluded that although the incidence of infection and hospitalization rates for COVID-19 were similar in their medical group compared to the matched Florida population (65 ), , the mortalty was $<0.05$ ) and $63 \%$ lower for those who were hospitalized $(p<0.05$ ) (Figure 2). Furthermore, they proposed that the introduction of their COVID-19 specific Population Health Program (PHP) was highly correlated to mortality reduction $(r=0.93, p<0.001)$.

The team believes that the improved outcomes were primarily due to two factors. First, an increase in patien engagement in the form of clinical encounters, home visits, and other direct communication. Second, the optimization of medical managemen hrough evidenced based treatment of chronic conditions. These findings were an extension of their stellit across a range of other clinical outcomes for the elderly by virtue of individualized care. Their evidencedbased process rapidly grew Cano Health from a single clinic to $>70$ primary care facilities staffed by $>175$ clinicians in four years.

\section{OOR PROGNOSIS}

Risk factors for poor prognosis from COVD-19 include old age and preexisting conditions including diabetes, fobesity, hypertension, congestive hear lung disease.

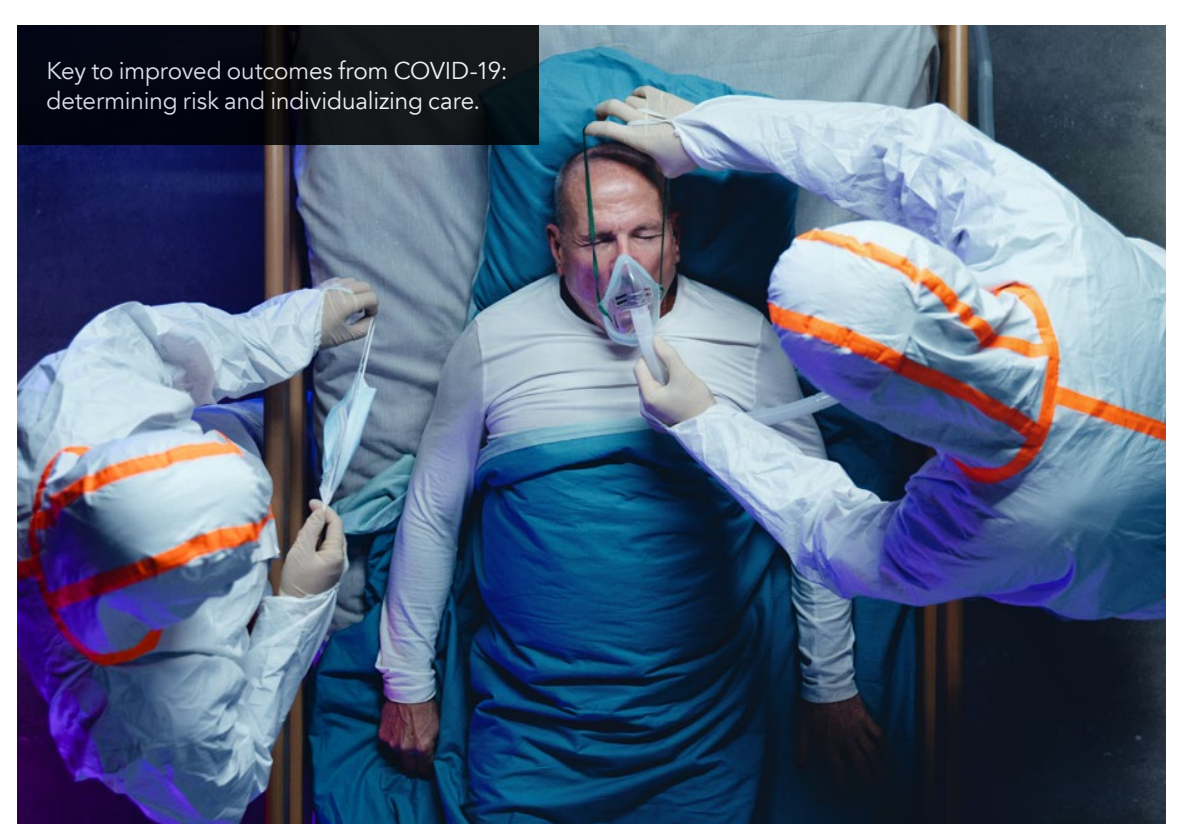

\title{
STAGES OF COVID-19
} The clinical course of COVID-19 infection consists of three clinical stages:

Stage I (Beginning of infection) Following an average incubation period of 5 days, most symptomatic symptoms such as experience mild fatigue, and body aches. These early symptoms usually last 5-7 days. During this time, the virus multiplies and becomes established in the respiratory tract of the host. Severity of symptom in Stage I correlate with the peak

Stage II (Pulmonary involvement) Some patients progress to Stage ll of

Stage III (Systemic inflammation) Only a small percentage of patients stage is associoted with infla. This throughout the body (extra-pulmonary systemic hyperinflammation syndrome). Unfortunately, the prognosis and recovery from Stage III is poor. Mortality is around $20-30 \%$.

To maximize the likelihood of recovery from COVID-19 infection, it is important to rapidly recognize the stage at which the patient is ill and treat them accordingly. However, as this is a new developed or standardized.

REVIEW OF EXISTING RESEARCH In April 2020, Dr Aguilar and his

$$
\text { colleagues from }
$$

Cano Health began

The work by Aguilar and his colleagues to tackle this new presents the first evidence-based challenge in care. In recommendations for an individualized individualized treatment for COVID-19. $\begin{aligned} & \text { care process across } \\ & \text { their clinics, they }\end{aligned}$ derived the first

the virus. Stage Il is characterized by a decrease in viral levels and an increase
in localized inflammation within the lungs. A computerized tomography (CT) scan or chest x-ray would show infiltrates (airspaces that become filled with other bodily cells or fluids) in the lungs and give the appearance of increased soft tissue density. This stage Stage I (5-7 days). evidence-based recommendation of individualized treatment from existing COVD-19 reports. These are essential given the variability of patient clinical presentation and survivability.

A literature search was conducted to find all relevant studies related to COVD-19. The search dates were to April 13, 2020, using the following 


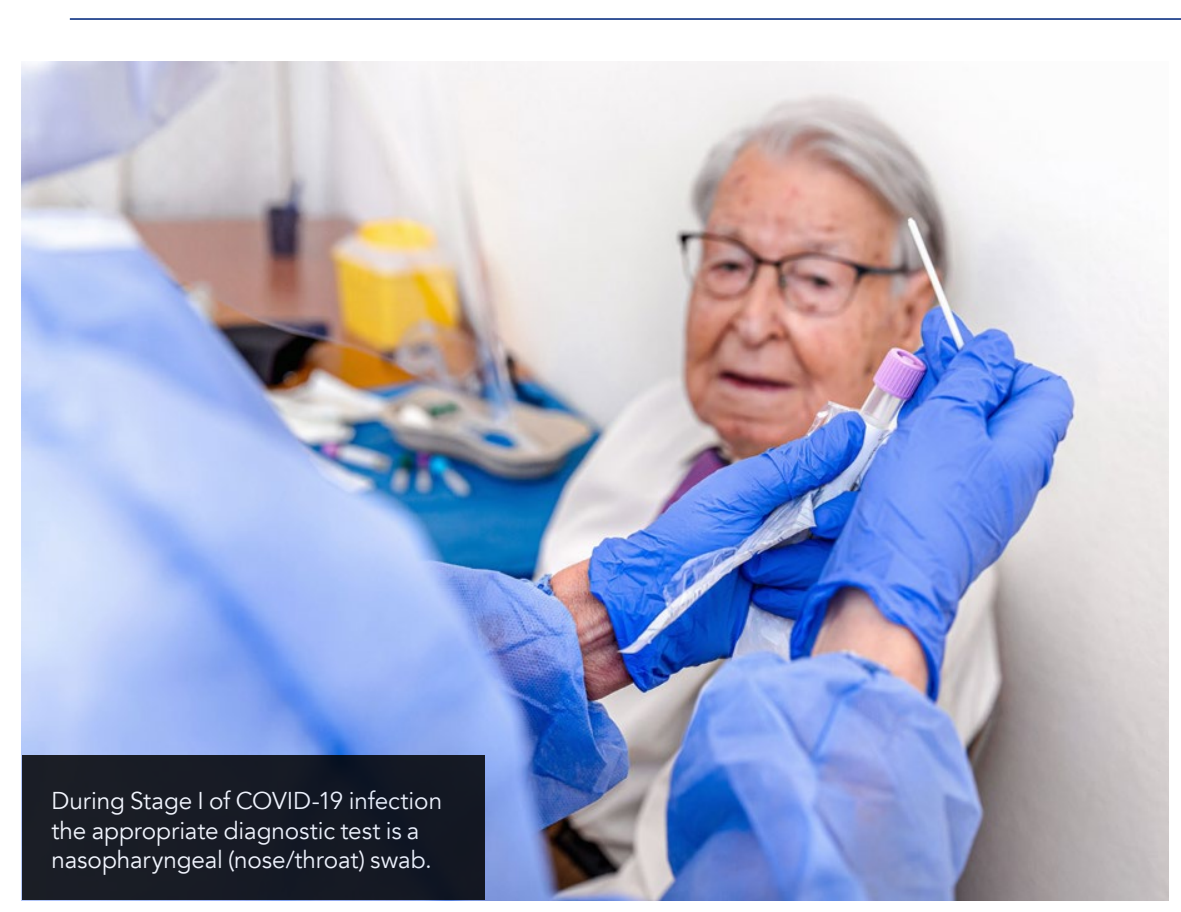

electronic databases: PubMed (1809 to present); Google Scholar (1900 to present); MEDLINE (1946 to pres

Embase (1980 to present). Aguilar and his colleaques located and reviewed 70 relevant studies on COVID-19 that were stored in medical and scientific databases. Their recommendations also drew upon guidelines from hospitals and clinics instructing their staff on procedures to follow. In addition, some decisions were based on data collected by organizations including the CDC, FDA, IHME, IDSA, and Worldometer. The largest group of participants numbered $<500$ and 30 patients.

The researchers focused on the following aspects of COVID-19 management: staged progression, clinical algorithms and individualized treatment. Based on their systematic review, Aguilar and colleagues created a model for the clinical stages of COVID-19, diagnostic tests, disease The results focused on providing individual treatment recommendatio by matching the most appropriate drug or treatment action for a given patient. For some patients, the treatment algorithm finds that no treatment is warranted; for others, treatments were based on age, preCOxing 19 symptoms.

INDIVIDUALIZED TREATMENT In Cano Health's model, disease of infection, extent ofinflammation and survivability of the patient The individualized treatment plan for COVID-19 first considers age and preexisting medical conditions. Second, the severity of COVID-19 symptoms and stage of infection. It also includes provision to update the treatment plan as patients progress through the stages of COVID-19 infection.

Stage I - During Stage I, the appropriate diagnostic test is a nasopharyngeal (nosed

Patients in Stage IIB are described as hypoxenic (2. Th abnormally low levels of blood oxygen). Patchts in Stage IIA are blood oxygen. The distinction between Stage IIA and IIB is important: in Stage IIB, the patient is likely to have difficulty breathing and corticosteroids (e. dexamethasone) may be considered. Oxygen supplementation care is given if needed, or anti-inflammatory treatments (e.g., colchicine). The use of these medications depends on age and preexisting health conditions.

Stage III - In Stage III, the most importan symptom is respiratory distress. Patients may have shortness of breath, rapid breathing, and have a bluish tinge to their skin. Treatment may include antivif medication in the presence of a high anti-congunts medications should be considered.

Some clinical trials have also explored pre-exposure and post-exposure prophylaxis (preventative antiviral treatment), but these approaches await more definitive research on their efficacy.

\section{EVIDENCE-BASED}

\section{TREATMENT IN PRACTICE}

The results of their literature review emphasized the patient treatment options to their baseline level of risk along with their stage of COVID-19 infection. Clinicians at Cano Health put this into practice to improve COVD-19

The team applied their clinical and population health tools to attempt to bend the curve and improve clinical outcomes.

The appropriate treatment is generally elderly Latino patients, many of
supportive care or antiviral medication.
whom have one or more pre-existing supportive care or antiviral medication Treatment must still be individualized based on age and pre-existing health conditions.

Stage II - There is a decline in viral load during Stage II, so treatment with antiviral medication is less effective than Stagel. Stage ll can be divided into two the oxygen levels in the patient's bon health conditions.

Dr Aguilar's group conducted a retrospective cohort study comparing managed care patients with an ageand gender-matched mirror group They iden compiling medical records, pharmacy They identified high-risk patients by

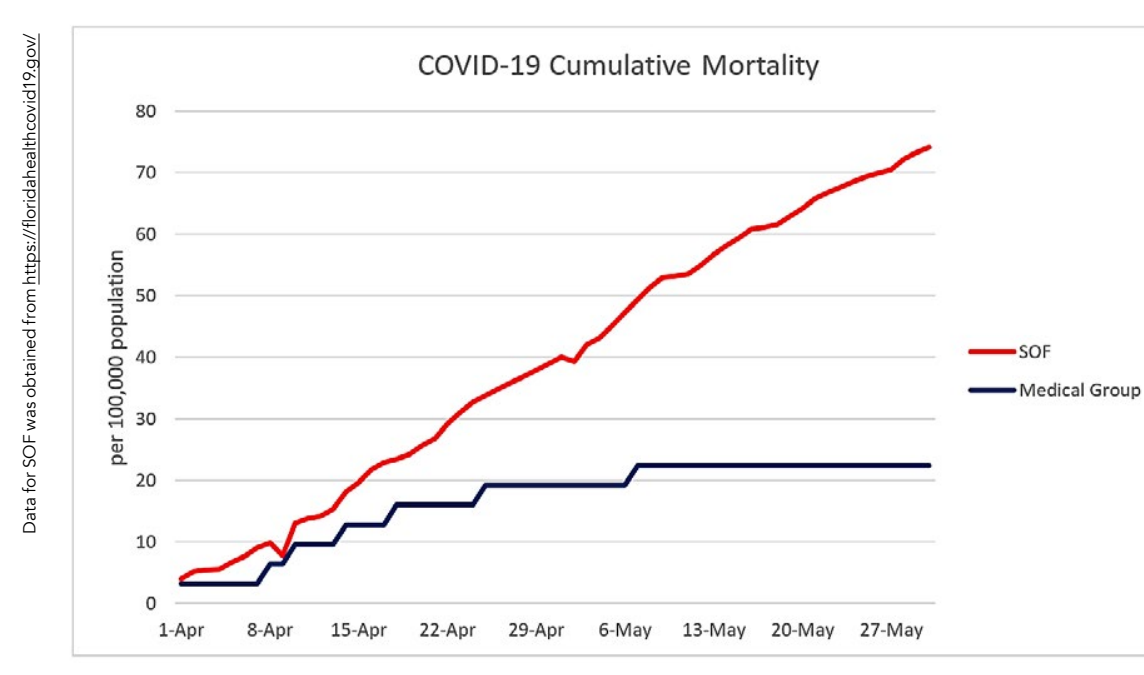

Fig2. COVID-19 Cumulative Mortality between Medical Group and SOF.

other medical statistics from their electronic medical records (EMR) plattorms, which readily provide total of 38,193 patients at high. A for COVID-19 were monitored for linical outcomes including COVID-19 cidence, hospitalization and motality The PHP involved contacting patients conducted medical encounters, and tracked data including comorbidities, treatment response and medical for publication, Managed Cacepted Health Program, Am $J$ Manag Care. 2021: Vol 27(6) P. 294 300: In Press. Feb 2021

In Cano Health's model, disease patients who declared symptoms of COVID-19 were and enrolled into the individualized treatment plan. Within Cano Health

progression was related to the level of infection, extent of inflammation and survivability of the patient. clinics, the highest risk patients (those
diagnosed with COVID-19 or at high diagnosed with COVD-19 or at high by the Medica Grou's cOVID Task Force (CTF), which cos Covid Task

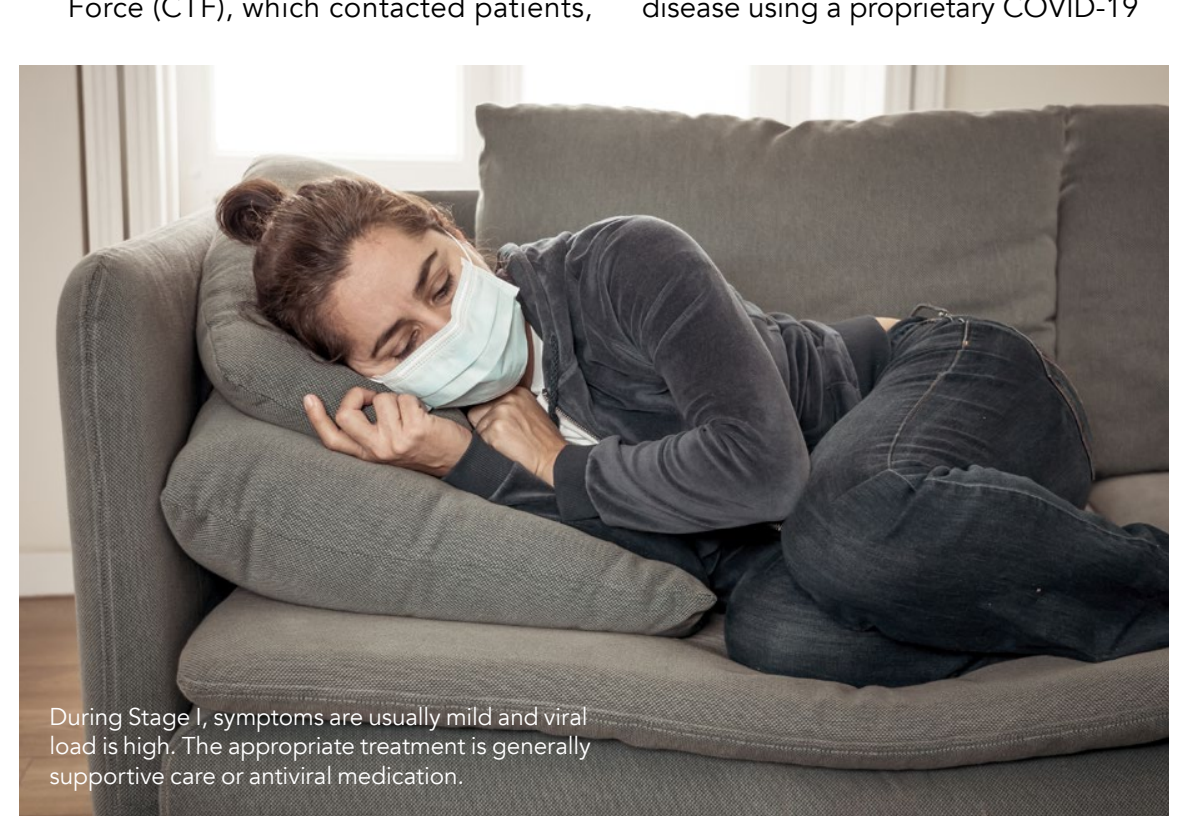

By implementing the Medical Group's COVID-19 PHP, patients for COVD-19 and/or underlying were diagnosed and treated early

survey. The COVID-19 survey was designed by the Medical Group's certifications in Internal Medicine and Family Medicine. The survey scores ranged from 0 to 162 based on age pre-existing comorbidities, current symptoms, length of illness, testing and diagnostic status for COVID-19, infection status of household members, current treatment, availability underlying medical conditions), and experience using Telemedicine. Patients were then sorted into one of four risk groups based on the score: low (0-10), high risk (11-29), high (30-40), and very $\geq 30$ or who we Patients with a score were assigned to the COVID-19 CTF. The CTF was a team of clincis CTF. other healthcare professionals assigned to manage patients. The CTF utilized a

inical team based on the findings

of their previous study. The patient's health status was continually monitored and updated, and treatment of underlying comorbidities optimized. The mean age of the Medical Group's samples

was 67.9 years \pm 15.2 years, $61 \%$ female and $39 \%$ male. Among their managed care patients, older age and hyctors predicting COVID-19. Obesty . hert failure were linked to higher rates of hospitalizations. After the initiation PHP) on April 6, the incidence of COVID-19 related hospitalization for patients in the Medical Group declined significantly by $63 \%$ ( $49 \%$ vs $28 \%$, p< 0.05 ) (Figure 3). They also reported a significantly reduced risk in mortality $60 \%(9.1 \%$ vs $1.6 \%, p<0.05)$ compared to the mirror group leading them to care was strongly associated with roved outcomes in Cano Health's large high-risk patient population.

A correlation was found between CTF A eferrals and overall mortality reduction 


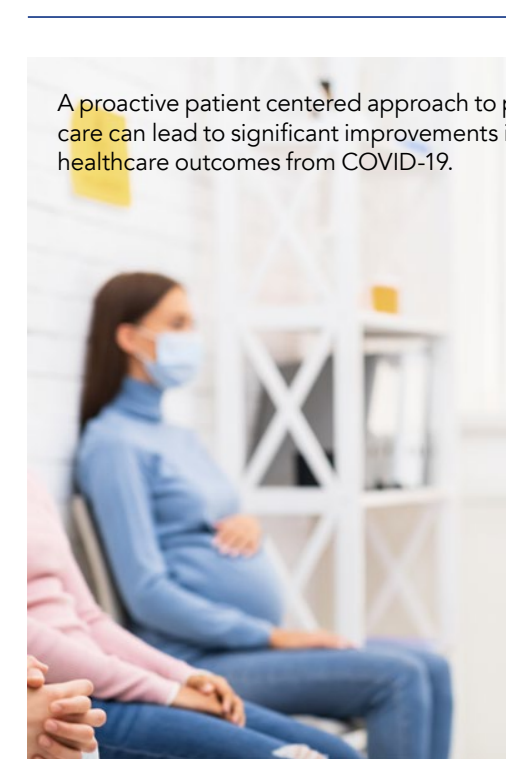

Florida mirror group over time $(r=0.93$ $p<0.001$ ) (Figure 4). The mortality reduction was found to be significant after treatment and all gender, outpatient In addition, they put forth the idea that the introduction of their COVID-19 specific PHP was highly correlated to mortality reduction $(r=0.93, p<0.00)$.

The work by Aguilar and his colleagues presents the first evidence-based recommendations for individualized treatment for COVID-19. Their reduced hospitalization and mortality data was published by the American Journal of Managed Care. These findings are limite by the flux of recommendations as the medical community learns more about in the right direction.

Their results suggest that a comprehensive PHP within the contex of managed care, in which highest risk patients are identified and treated early may significantly reduce mortality from COVID-19. This was true for their senior population, as compared to age and gender Florida matched controls. Although they used several off-label treatments, this study was not powered to evaluate the effect of any particular treatment modality. Moreover, their program also included several addition outpatient interventions, such as the delivery of pulse oximeters and oxygen concentrators to the patient's home. This outpatient intervention may have provided additional clinical benefit through selection
setting of care.

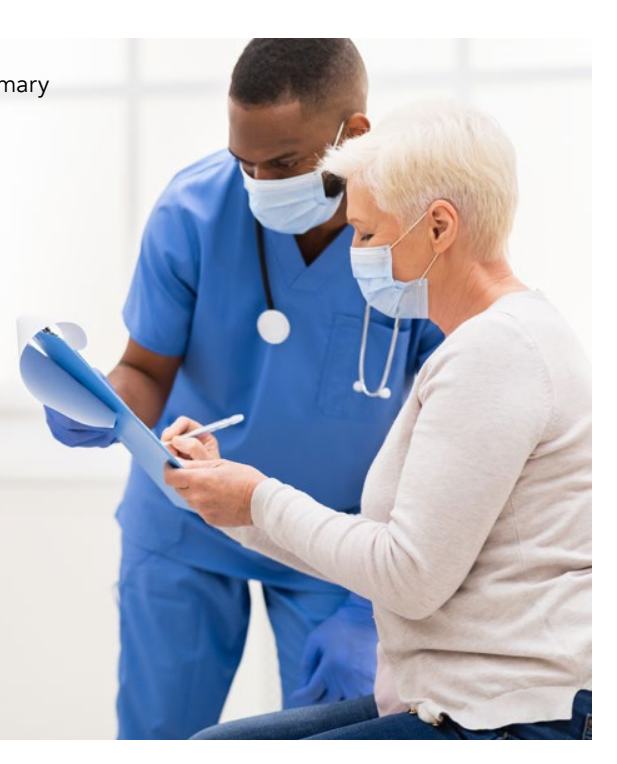

In addition, their study adds to a growing body of work concluading that the deliven from episodic care to direct emptive care can result in significant benefits This narrative is especially critica in low socioeconomic circumstances, where morbidity and mortality tend to be higher and where access to care is a major barrier to improving health outcomes. COVID-19 has presented monumental challenge to our healthcare system and the high incidence and complexity of this disease requires a paradigm shift in our healthcare delivery from reactive to a targeted proactive approach. This approach should be led by primary care providers who work on

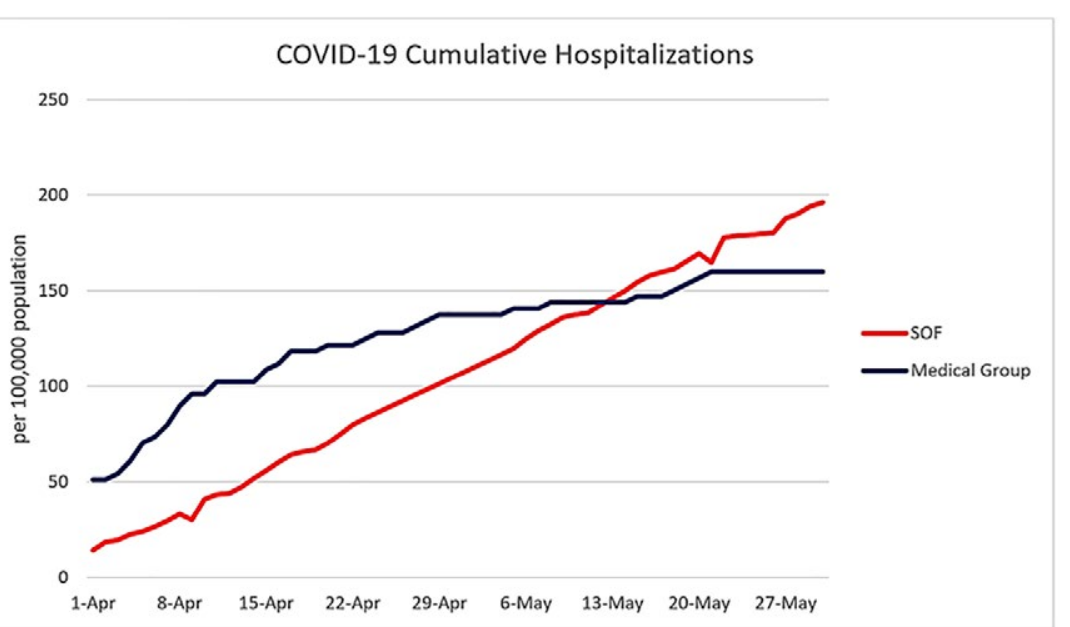

1-Aer 15-Apr 22-Apr 29.Apr 6. May 13-May 20-May 27-Mar Fig3. COVID-19 cumulative hospitalizations between Medical Group and SOF.

This data suggests that the delivery of a proactive patient centered approach to primary care can lead to significant

the front line in conjunction with targeted state of the art population health data and the implementation of optimized improvementsin hel case standards of care
chronic diseases.

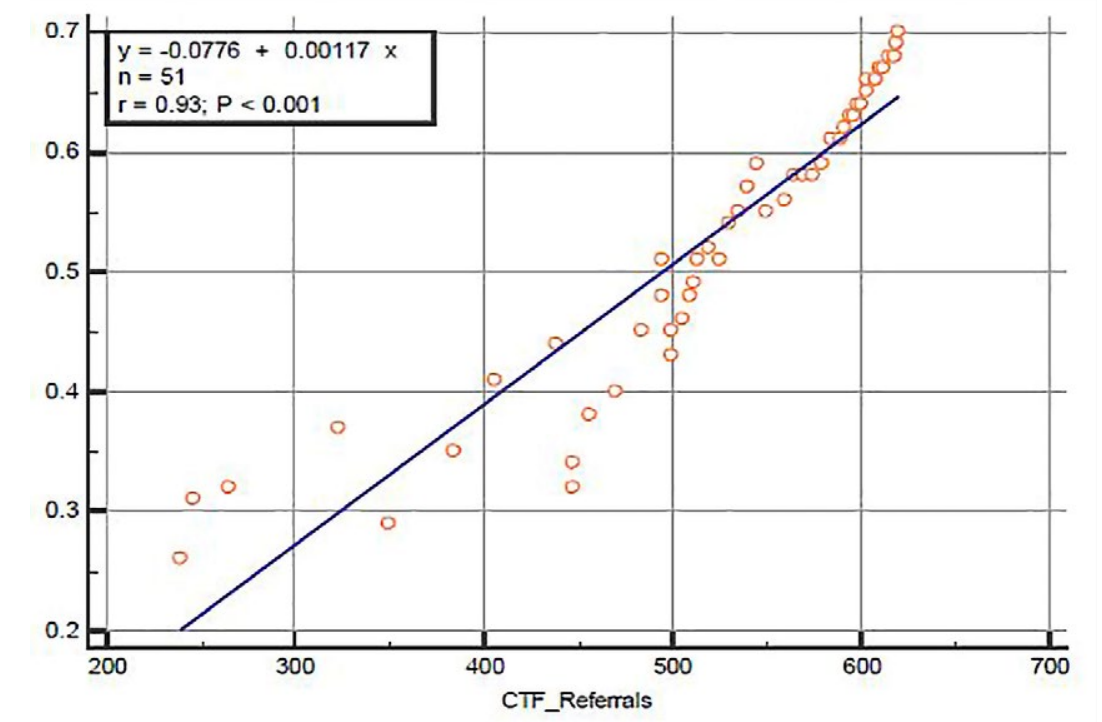

Fig4. Overall mortality reduction and COVID Task Force (CTF) referral correlation.

\section{Behind the Research}

Richard B. Aguilar, MD

E: Richard.aguilar@canohealth.com T: +1 (562) 896-7969

Lina Narvaez, Administrative Assistant E: lina.narvaez@canohealth.com T: +1 (754) 244-5099 @ @canohealthfl $⿴ 囗 十)$ https://youtube.com/channel/UCj1DSxZvdno-icfoS1DHBUA

\section{Research Objectives}

Cano Health's mission is the triple aim, improve patien forging a life-long bond with members while reducing costs.

\section{Detail}

Address

Dr Richard B. Aguilar, Chief Clinical Officer Cano Health, LLC, 9725 NW 117 Ave, Suite 200

Miami, FL. 33143. USA

Richard B. Aguilar, MD, is an Internist and the $\mathrm{CCO}$ of $\mathrm{Can}$ Health, LLC based in South Florida. He helped grow Cano Health from 1 clinic to $>75$ offices across 4 states. Inc. 5000 selected Cano Health as the fastest growing primary care in the US in 2018 and 2019.

\section{Collaborators}

ceria, MD

Patrick Hardigan, PhD

- Giorgio Tarchini, MD

- Darby Sider, MD

- John McGoohan, DO

- Merlin Osorio, MD

- Kathleen Hagen, EdD

Marlow B. Hernandez, DO, MPH

- Bianca Barrow, MBA, CSSBB

Special thanks to Pedro Cordero, Toni Mikell, and the Cano Health Population Health and Care Management teams who recorded detailed patient-level data and coordinated the COVID-19 Task Force program. Without them, this work

\section{References}

- Siddiqi, H. and Mehra, M. (2020). COVID-19 illness in native and immunosuppressed states: A clinical therapeutic staging proposal. J Heart Lung Transpl. [online] 39(5),
405-407. https://www.ncbi.nlm.nih.gov/pmc/articles/ PMC71186521

- Aguilar, RB. et al. (2021). Managed Care COVID-19 Outcomes in a Population Health Program. Am J Manag Care. 2021;27(6): In Press. https://www.ajmc.com/view/ managed-care-covidh

\section{Personal Response}

\section{What is the next priority in COVID-19 research?}

II Managing patients during the post COVID-19 infection period. Especially those with lingering
symptoms of pulmonary compromise (continued use of oxygen and shortness of breath) and mental complications which may lead to prolonged confusion and lack of concentration (i.e. poor lung function and
cognitive declines).

\section{What are the best tools for following these patients}

II Incentive spirometry metrics, such as: how to manage patients with low $\mathrm{FiO}_{2}$, use of inhaled steroids Tracking the longevity of the resulting immunity as well as possible re-infection rates that we are now seeing and being reported. Dealing with the possibility of yearly COVDstandardized outpatient treatment regimens. Early standardized ourpation treatuce tregimens. Early
treatment intervention to reduce the inmatory complications resulting from the cytokine storm
eventually leading to blood clotting disorders, respiratory distress and multi-organ damage.

\section{Are there similar treatment frameworks for othe}

II There are other diseases that result from an overactive inflammatory diseases. Interestingly steroids and hydroxychloroquine are used (or have been proposed) for the treatment of both COVID-19 infection and rheumatologic autoimmune disorders. Commenting on rheumatologic disorders in children, Dr Randy Cron,
a pediatric rheumatologist and immunologist at the a pediatric rheumatologist and immunologist at the
University of Alabama at Birmingham and co-editor of the 2019 textbook Cytokine Storm Syndrome. "They tend to articl/

Early evidence, again from China, indicates that the antibody tocilizumab (Actemra) may be beneficial for COVID-19. cytokine, preventing cells from receiving the IL- 6 mess Tocilizumab is normally used to treat arthritis and to alleviate cytokine storms in cancer patients receiving immunotherapy. 